\title{
Tecnologia Assistiva e Neurodegeneração: Estimulação Cognitiva com Jogos Digitais
}

\section{Assistive Technology and Neurodegeneration: Cognitive Stimulation with Digital Games}

\author{
Márcia Cristina Domingues Leite ${ }^{1}$ \\ Maria Elaine dos Santos Leon ${ }^{2}$
}

\begin{abstract}
Resumo: O grande aumento da expectativa de vida do brasileiro nas últimas décadas acende um alerta para um olhar mais voltado para a pessoa idosa. Sabe-se que nem sempre a idade vem acompanhada de uma boa qualidade de vida, e para tanto se faz necessário que as mais diversas áreas do conhecimento se sensibilizem com este importante nicho populacional, entregando propostas que visem seu bem-estar. $O$ presente estudo apresenta o desenvolvimento de um protótipo de um ambiente virtual com jogos para estimulação cognitiva para pessoas com neurodegeneração proveniente do processo natural de envelhecimento. Através de uma pesquisa descritiva e exploratória foi possível estudar tais doenças e seus sintomas para então desenvolver o protótipo, ao final foi feita a avaliação da usabilidade com idosos do Centro do Idoso da cidade de Bagé/RS, onde o protótipo obteve um grau de usabilidade de $83 \%$ e a partir desse índice e dos demais resultados será possível implementar melhorias que contribuirão para entregar um sistema altamente usável e útil para o usuário final.
\end{abstract}

Palavras-chave: Doenças neurodegenerativas; Jogos digitais; Reabilitação cognitiva.

\begin{abstract}
The great increase in the life expectancy of the Brazilian in the last decades warns for a more oriented towards an elderly person. It is known that not always age comes accompanied by a good quality of life, and what is necessary to make you know more areas of knowledge is sensitized with this important population niche, delivering proposals for your well-being. The present study presents the development of a prototype of a virtual environment with games for cognitive stimulation for people with neurodegeneration coming from the natural process of aging. Through a descriptive and exploratory research to study these diseases and their symptoms for the development of the prototype, at the end an evaluation of the usability was made with the elderly of the Center of the Elderly of the City of Bagé / RS, where the prototype obtained a degree of usability of $83 \%$ from the index and other results will be possible to implement improvements to the project for a highly useful and useful system for the end user.
\end{abstract}

Keywords: Neurodegenerative diseases; Digital games; Cognitive rehabilitation.

\footnotetext{
${ }^{1}$ Bacharel em Sistemas de Informação URCAMP, Campus Bagé - Av. Tupy Silveira, 2099 - Bagé (RS) Brasil

\{marcialeite@urcamp.edu.br\}

${ }^{2}$ Mestre em Geoprocessamento URCAMP, Campus Bagé - Av. Tupy Silveira, 2099 - Bagé (RS) - Brasil

\{marialeon@urcamp.edu.br\}

Rev. CCEI - URCAMP, V.23, n38 2018 (Submetido 02/07/2018; Aceito: 25/09/2018)

https://doi.org/10.30945/ccei-v23i38.343
} 


\section{INTRODUÇÃO}

A sociedade passa por constantes transformações nos mais diversos contextos principalmente no que diz respeito à evolução tecnológica, que viabiliza diariamente uma gama de recursos que facilitam e modificam sobremaneira a vida das pessoas. Tais dispositivos antes utilizados apenas para realizar operações numéricas complexas, hoje servem como ferramenta de grande funcionalidade nos mais diversos contextos, dentre os quais é possível destacar seu uso como recurso terapêutico no campo da saúde (WANATABE, 2003).

A utilização de tecnologias em consonância com outras ações de caráter interdisciplinar e que possibilitam que pessoas com algum tipo de deficiência consigam realizar atividades antes julgadas impossíveis conceituam o termo Tecnologia Assistiva (TA). Uma de suas principais propostas é justamente melhorar a qualidade de vida de grupos específicos de pessoas com diferentes deficiências físicas e/ou cognitivas (OLIVEIRA, 2015).

Na população brasileira, o grupo social cuja a faixa etária mais cresce são os idosos (IBGE, 2015), e esses números tendem a aumentar, pois de acordo com a Tábua de Mortalidade publicada no Diário Oficial da União (DOU) pelo Instituto Brasileiro de Geografia e Estatística (IBGE, 2015), a expectativa média do brasileiro é de 75 anos. Ainda segundo o Instituto, a expectativa média de idade dos brasileiros deve aumentar para 81 anos, sendo que as mulheres continuarão tendo uma expectativa de vida maior que os homens chegando em 2060 a 84 anos enquanto a dos homens não passará dos 78 anos.

Tais números sugerem um olhar mais voltado para o cuidado da pessoa idosa tendo em vista o comprometimento de sua saúde através do processo de senilidade. Estudos demonstram que o processo natural de envelhecimento traz consigo uma inexorável degeneração de diversos órgãos, que resultam em limitações expressivas que refletem na qualidade de vida dos indivíduos. Uma das doenças que mais acometem idosos são as de natureza crônico-degenerativa, dentre as quais estão as doenças neurodegenerativas. Isso porque, à medida que se envelhece, é natural que o comportamento de um modo geral e principalmente o cognitivo sofra alterações e até mesmo perdas significativas como dificuldade de memorização, diminuição da autonomia e consequentemente da qualidade de vida como explicam Ávila e Miotto (2003). 
O Manual de Diagnóstico e Estatística de Transtornos Mentais (DSM-IV, 1994) da American Psychiatric Association , define a demência como um processo caracterizado pelo declínio da memória aliado ao deficit de pelo menos uma das diversas funções cognitivas com uma intensidade capaz de interferir sobremaneira no desempenho social e profissional dos indivíduos.

Existem neurodegenerações irreversíveis, como a Doença de Alzheimer (DA), que por sua vez, possuiu maior incidência de casos (HAMDAM, 2008). Já as neurodegenerações reversíveis provenientes do processo natural de envelhecimento ou de outras doenças geralmente afetam o Sistema Nervoso Central (SNC), acarretando alterações funcionais, celulares e deterioração dos neurotransmissores, que são os principais "mensageiros" do cérebro, fazendo com que diminua a capacidade cognitiva e motora da pessoa ao longo do processo degenerativo (FORNARI et al., 2010).

Dentro do campo de Tecnologia da Informação (TI) é possível propor estratégias de inclusão da pessoa idosa junto as novas tecnologias do mundo contemporâneo, assim como também é possível utilizar as mesmas como recurso terapêutico de auxílio no tratamento de diversas doenças, que neste caso, foi o foco deste estudo. Concomitantemente ao tratamento medicamentoso, fisioterapêutico e psicológico é possível que outros recursos auxiliem a retardar essa neurodegeneração. É justamente neste ponto que os jogos surgem como uma proposta de auxílio terapêutico na reabilitação cognitiva destes indivíduos. Eles são uma alternativa na recuperação das funções cognitivas, sensório-motoras, deficiências neuropsicológicas, pois servem como exercícios terapêuticos uma vez que oferecem a possibilidade de integração cultural aos usuários (WANATABE, 2003).

A partir do exposto, o estudo teve como objetivo desenvolver um protótipo de ambiente virtual com jogos no intuito de auxiliar pessoas com doenças neurodegenerativas provenientes do processo natural de envelhecimento em sua reabilitação cognitiva na cidade de Bagé/RS.

O estudo foi estruturado da seguinte forma: a seção 2 contemplou o referencial teórico, que abordou todo o embasamento científico sobre as doenças neurodegenerativas, principalmente as reversíveis e que serviu com requisito para o desenho e formatação do protótipo. Já a seção 3 demonstra toda a metodologia utilizada e as etapas realizadas até a 
conclusão do estudo e alcance do objetivo geral. O item quatro mostra os resultados, onde constam detalhadamente todas discussões envolvendo, desenvolvimento, avaliação e a resposta dos usuários ao uso da ferramenta. Finalmente, a seção 5 apresenta as considerações finais, onde foi feita a síntese das conclusões do estudo.

\section{ABORDAGEM TEÓRICA}

No decorrer dos anos, jogos têm sido concebidos não somente para o entretenimento, mas também para outros fins como por exemplo a aprendizagem, marketing e também práticas terapêuticas de saúde (ARAÚJO et al., 2010), e se mostraram eficientes no que diz respeito ao uso da população idosa principalmente pelo fato de proporcionarem o aumento do desempenho na fluência e na percepção visual de tais indivíduos (WHITLOCK et al., 2012).

Costa (2005) e Rieder (2005) explicam que jogos digitais desafiam o usuário e ao serem resolvidos, estimulam diversas funções cognitivas, como por exemplo a atenção, a memória e a concentração. Ainda sobre a cognição, eles estimulam a atenção, concentração e memorização e também demonstram eficiência no lado físico ao proporcionarem o aprendizado motor ao despertarem a capacidade de planejar as ações, como afirma Corrêa et al. (2008).

A Organização Mundial de Saúde (OMS, 2014) define a reabilitação cognitiva como um conjunto de medidas que proporcionam que indivíduos que experimentam alguma incapacidade alcancem ou mantenham sua interação com o meio em que vivem. Ela prima pela independência destes indivíduos e permite que eles levem uma vida normal dentro do meio familiar, social e de trabalho. Xavier (2016), em seu estudo acerca das doenças neurodegenerativas complementa o exposto anteriormente quando sugere a inclusão digital como ferramenta para promover a reabilitação cognitiva de pacientes em fase inicial de processos demenciais, para tanto, ele afirma que até mesmo pessoas em estágios iniciais de Doença de Alzheimer “são mais capazes de aprender do que antes se pensava”. Ainda segundo ele, tais práticas são apropriadas não só para idosos com diagnóstico mais graves como de DA, mas também para aqueles que já apresentam Comprometimento Cognitivo Leve (CCL). O autor ainda esclarece que recentes estudos já demonstraram a eficácia da reabilitação cognitiva em pessoas em fase inicial de processos demencial, visto que estes 
apresentaram melhoria no que diz respeito a sua orientação de tempo e espaço em comparação a grupos de pessoas no mesmo estágio demencial e que não receberam nenhum tratamento de reabilitação.

Rocha et al.(2014) afirma que psicólogos tanto no cenário nacional como no internacional demonstram grande interesse em suas investigações acerca das contribuições dos jogos digitais no sentido de desenvolver habilidades como a flexibilidade cognitiva, atenção seletiva e sustentada, etc. Tal ideia já era considerada por Lévy (1999) quando este afirmava que as tecnologias reorganizavam e modificavam o campo cognitivo das pessoas envolvidas, uma vez que possibilitava reorganizar algumas funções cognitivas como memória, atenção, criatividade e que possibilita sobremaneira verificar o modo de percepção e intelecção que cada pessoa possui sobre determinado contexto.

\subsection{USABILIDADE}

No que diz respeito a usabilidade, esta possui uma definição simples e objetiva descrita por Nielsen e Loranger (2007) ao definirem-na como um atributo que entrega facilidade de uso a algo. Eles se referem à rapidez com que o usuário aprende algo, sua eficiência ao usar um sistema, o quanto lembram dele e o quanto gostam de usá-lo.

A norma ISO 9241 (International Standards Organization) define a usabilidade como um padrão de medida que verifica se um produto pode ser utilizado por usuários específicos com eficácia, eficiência e satisfação dentro de um contexto (ISO, 1998). Pressman (2011) em uma de suas obras afirma que a usabilidade é uma das principais características para que uma interface consiga atingir um nível satisfatório de interação entre o humano e a máquina, uma vez que, através desta o usuário cadencia seu ritmo de trabalho e alcança seu objetivo através do uso da aplicação.

John Brooke (1986), em seu estudo acerca da usabilidade enfatiza que não existe apenas uma maneira de verificar se um sistema tem um alto grau de usabilidade, visto que ele deve ser coerente com o fim a que se destina.

Nielsen (1993), um dos precursores nos estudos sobre design de interface do usuário, elencou dez heurísticas para serem usadas no projeto de uma interface com foco na qualidade 
da interação e experiência do usuário. Tais heurísticas devem nortear o projeto no intuito de que seja possível a utilização da ferramenta pelo maior número possível de usuários.

\subsection{AVALIAÇÃO DA USABILIDADE}

Paz e Leite (2017) em seu recente estudo acerca da avaliação da usabilidade utilizaram o questionário SUS para medir o grau de usabilidade de uma aplicação e explicam que o documento é constituído por dez perguntas respondidas através de marcação de uma das alternativas da escala Likert, conforme também afirmam Boucinha e Tarouco (2013). Esta escala, de acordo com Mafra (1999), "mostrará o grau de concordância e/ou discordância do usuário em relação aos elementos avaliados", e como é demonstrado na Figura 1.

Figura 1: Escala Likert SUS.

\begin{tabular}{|c|c|c|c|c|c|}
\hline & $\begin{array}{l}\text { Discordo } \\
\text { Totalmente }\end{array}$ & & & & $\begin{array}{l}\text { Concordo } \\
\text { Totalmente }\end{array}$ \\
\hline & 1 & 2 & 3 & 4 & 5 \\
\hline
\end{tabular}

Fonte: Adaptado de Brooke (1986).

\section{METODOLOGIA}

O estudo foi desenvolvido da seguinte forma: primeiramente foi feita uma pesquisa bibliográfica de caráter descritivo e exploratório em que se buscou embasamento científico sobre o assunto pertinente ao trabalho, necessário para análise e compreensão do estudo (PRODANOV e FREITAS, 2013). Foi utilizado o acervo de documentos científicos disponíveis na internet e em livros onde o foco foram as doenças neurodegenerativas e de que forma elas afetam os indivíduos. Isto serviu como base para projetar quais os jogos poderiam auxiliar de forma mais eficaz os pacientes.

A etapa seguinte foi o desenvolvimento do ambiente virtual, conduzido segundo o modelo de software de prototipação. De acordo com Beyer e Holtzblatt (1999) e Engholm (2010), protótipos permitem a constante comunicação entre o designer e os clientes, agregando troca de experiência, o que contribuiu significativamente para um desenho mais apropriado às necessidades do projeto. Esta metodologia se deu com a incorporação de cinco passos distintos como segue: 
1. Comunicação (coleta e refinamento de requisitos): Uma das primeiras etapas para a construção de um projeto de software é saber o que será necessário para o desenvolvimento. Nesta etapa foram definidos os jogos que seriam desenvolvidos. Nesse sentido, Sommerville (2012) e Machado (2014) afirmam que os requisitos são o ponto de partida de qualquer projeto e que eles refletem as características e as restrições que o software deve ter sob o ponto de vista das necessidades do usuário e isso não leva em conta qual tecnologia foi utilizada para o desenvolvimento do mesmo.

2. Plano Rápido: Na sequência, ficou definido que a linguagem de programação a ser utilizada seria HTML5, CSS3 e JavaScript. Segundo Prado (2012), o HTLM5 permite que se desenvolva aplicações mais complexas sem necessariamente utilizar plugins e ainda ser utilizada em qualquer dispositivo com acesso à internet e que juntamente com a manipulação de folhas de estilos CSS e JavaScript, entregam uma interface intuitiva e moderna ao usuário (BRITO E ZANELATO, 2015). Com o auxílio da biblioteca Bootstrap, disponibilizada no Github e no Wordpress com código aberto é possível desenvolver de forma mais rápida o layout da aplicação.

3. Modelagem Rápida: Nesse momento do processo ocorre a modelagem da aplicação. Através de ferramentas específicas de modelagem por padrões universais determinouse de que forma seria realizado o desenvolvimento do projeto.

4. Construção do Protótipo: Nesta fase o desenvolvimento foi efetivamente realizado utilizando a linguagem de programação escolhida e de acordo com as informações modeladas na etapa anterior.

5. Implantação, entrega e feedback: O passo seguinte consistiu e testar a aplicação com idosos do Centro do Idoso da Prefeitura Municipal de Bagé/RS, entregando a eles os jogos e verificando sua interação com a aplicação de um modo geral. Ao final, todos responderam o questionário SUS( System Usability Scale) desenvolvido por John Brooke em 1986 conforme sintetizado na próxima seção deste estudo.

Finalmente foi possível mensurar o grau de satisfação dos usuários em relação ao protótipo desenvolvido e projetar trabalhos futuros com as melhorias sugeridas e com o 
intuito de disponibilizá-lo a pacientes portadores de processos neurodegenerativos auxiliandoos em sua reabilitação cognitiva. A Figura 2 ilustra uma adaptação do modelo de prototipagem de Pressman (2011) e entrega uma análise mais clara de todas as etapas do estudo descrito até então.

Figura 2: Modelo de prototipagem.

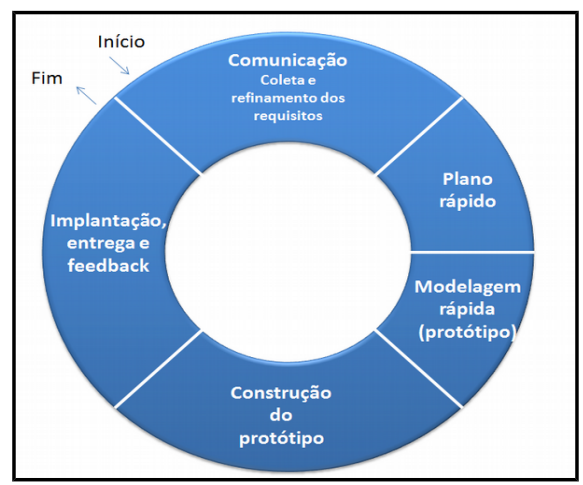

Fonte: Adaptado de Pressman (2011).

\section{RESULTADOS E DISCUSSÃO}

O desenvolvimento do protótipo para treino cognitivo contou com uma série de processos que permitiram estudar e entender as consequências do processo natural de envelhecimento no que diz respeito ao raciocínio dos indivíduos. A partir desse entendimento, foi possível projetar os jogos, o layout e as técnicas de acessibilidade utilizados de modo a entregar um sistema que possibilitasse uma boa interação humano computador, como pode ser verificado a seguir.

\subsection{DESENVOLVIMENTO DA INTERFACE E DOS JOGOS}

De acordo com o consórcio internacional World Wide Web (W3C, 2014), para que um site seja desenvolvido de forma acessível existe a necessidade de interconexão entre componentes. Essa conexão se refere ao conteúdo, códigos de marcação, ferramentas de criação, desenvolvedores e as tecnologias necessárias para a deficiência que está em questão. A W3C determina que independente da deficiência, um site possa ser utilizado por todos, ou seja, qualquer pessoa possa compreender e interagir com o conteúdo web disponível seja qual for sua limitação. 
O desenvolvimento da interface e dos jogos teve como fatores norteadores aspectos sobre a usabilidade voltada para a pessoa idosa. Para tanto, optou-se por simplicidade, possibilidade de ajuste do tamanho da fonte e uso adequado das cores de modo a compensar algumas habilidades perdidas por pessoas idosas durante o processo natural de envelhecimento.

Desenvolver uma interface simples e objetiva facilita o entendimento por parte do usuário, explica Leite (2014), tendo em vista que ele terá o desafio de lembrar ou aprender as regras do jogo escolhido. Outro detalhe importante utilizado no desenvolvimento dos jogos se relacionou ao tempo, pois jogos de curta duração proporcionam momentos agradáveis tanto para o paciente quanto para seu cuidador. No que diz respeito às cores, ao longo dos anos os indivíduos têm uma tendência a perda natural da sensibilidade ao contraste das cores e da percepção sobre as mesmas. Alguns detalhes como adaptação ao claro e ao escuro em relação a diferentes interfaces causa dificuldade no que diz respeito a interação da pessoa idosa (MENDES, 2008). Tais fatores influenciaram na escolha de cores mais escuras, bem como padronizar todas as telas do jogos com o mesmo plano de fundo no sentido de entregar conforto visual ao público-alvo do protótipo, conforme mostra a Figura 3.

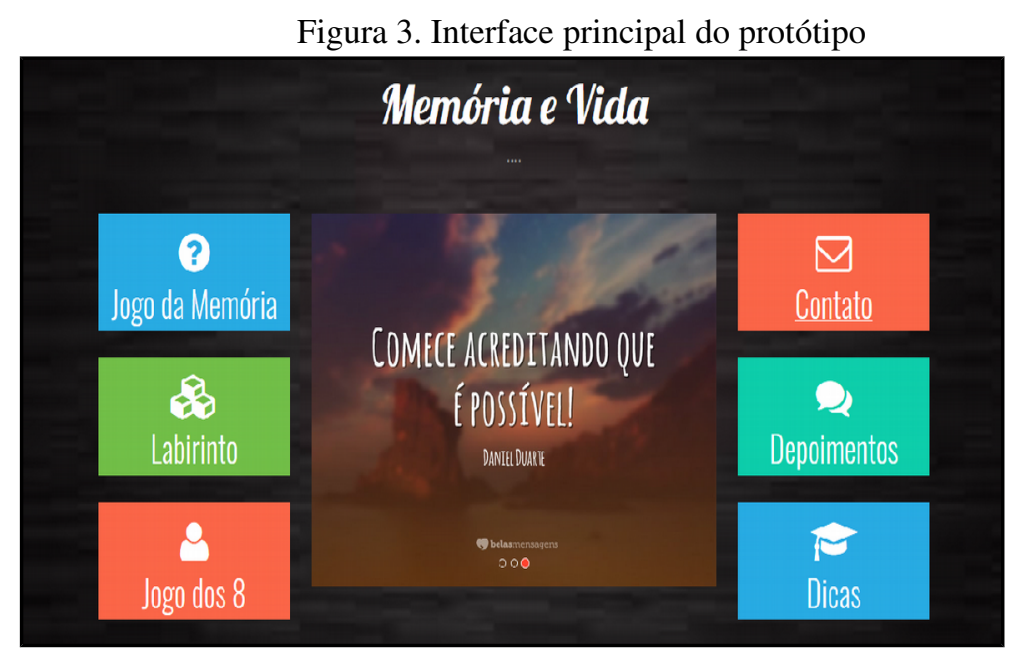

Fonte: Dados Primários (2017).

O atributo ALT foi utilizado no código, o que permitirá que leitores de tela façam o reconhecimento audio descritivo auxiliando usuários com limitações visuais a utilizarem o sistema (RIOS et al., 2016). 


\subsection{JOGO DA MEMÓRIA}

O primeiro jogo desenvolvido foi o Jogo da Memória que como o próprio nome diz teve como principal objetivo estimular a memória e o raciocínio do usuário. O jogo consiste em encontrar os pares de figuras virando as cartas com um click sobre elas. Nessa parte do projeto a prioridade foi centralizar o jogo e utilizar fontes de imagens grandes pois Nielsen e Loranger (2007) explica que os tamanhos reduzidos são um dos principais obstáculos encontrados por idosos durante sua interação em web sites, conforme Figura 4.

Figura 4: Jogo da memória.

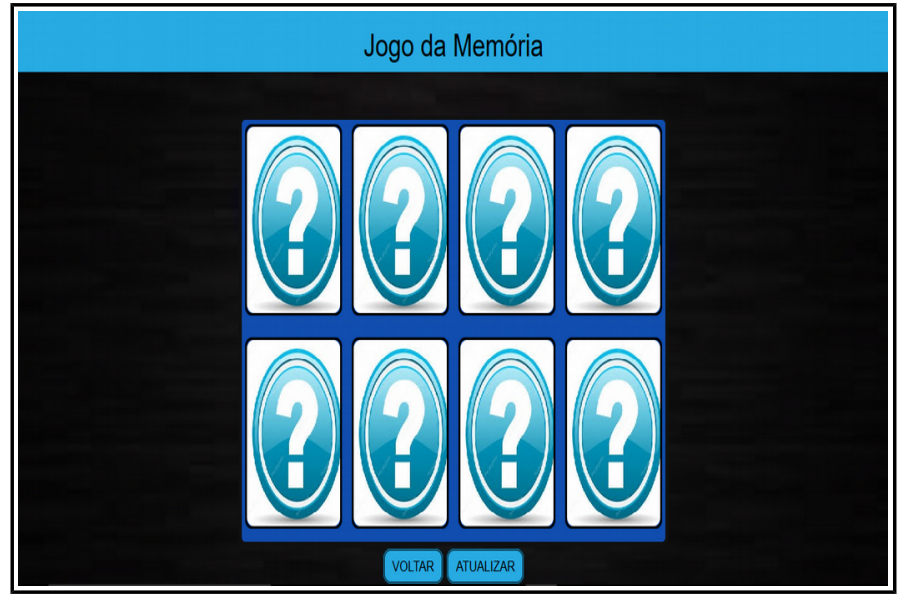

Fonte: Dados primários (2017).

\subsection{JOGO DO LABIRINTO}

O jogo do labirinto foi inserido dentro do projeto com o objetivo de treinar as funções executivas de um modo geral, dentre estas, despertar a capacidade de resolução de problemas dos usuários. Souza et. al (2006) explica que tal atividade permite avaliar aspectos neuropsicológicos importantes como por exemplo a organização espacial, o planejamento e a execução de quem joga, pois até alcançar o outro extremo do labirinto requer uma operacionalização de movimentos e planejamento de ações, portanto requer estratégias cognitivas dos jogadores. A ideia central do jogo consiste em encontrar uma forma de levar o personagem até o final do labirinto, conforme demonstra a Figura 5. 
Figura 5: Jogo do Labirinto.

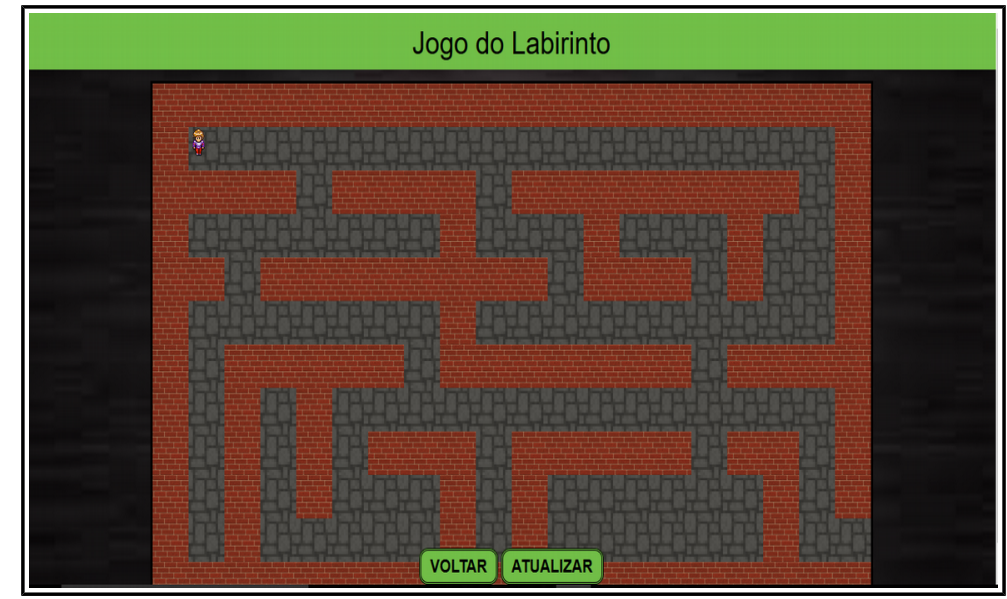

Fonte: Dados Primários (2017).

\subsection{JOGO DOS OITO}

O jogo dos oito consiste em organizar os números de forma lógica e sequencial, para isso o jogador deve mover as peças em um tabuleiro que possui apenas uma casa em branco. Esse jogo exige um planejamento e estratégia semelhante ao jogo do labirinto, porém apresenta um nível de complexidade muito maior, conforme demonstrado na Figura 6.

Figura 6: Jogo dos oito.

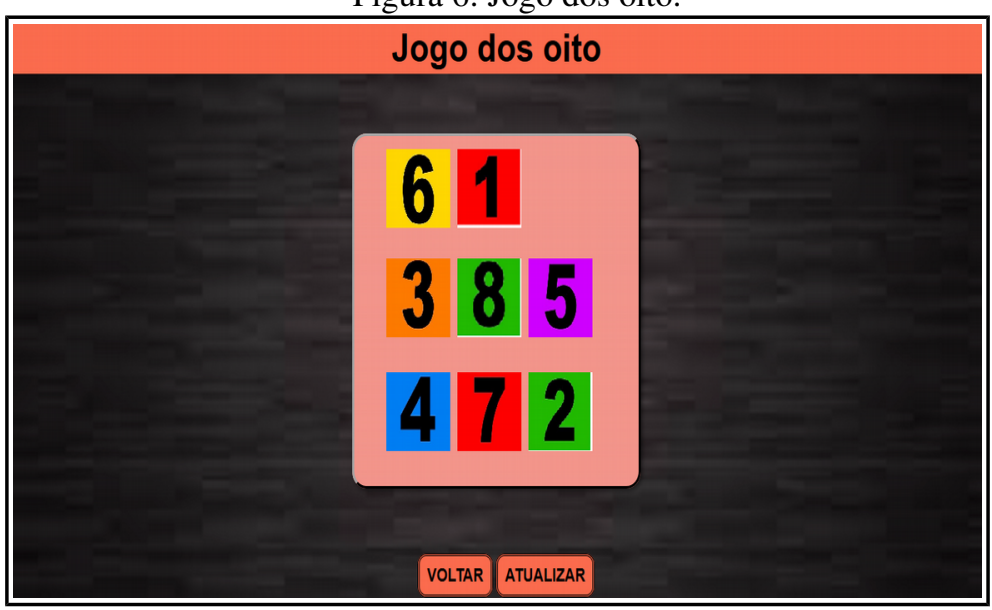

Fonte: Dados Primários (2017). 


\subsection{AVALIAÇÃO DA USABILIDADE}

Para realização da avaliação da usabilidade foi utilizado o questionário SUS conforme mencionado anteriormente. Os idosos utilizaram o protótipo durante a aula que acontece normalmente dentro das atividades propostas pelo Centro. No decorrer da aula, os mesmos foram observados utilizando o protótipo pois a observação é um aspecto determinante na análise dos resultados e na realização da tarefa, conforme afirma Nielsen (2003). O autor ainda complementa que a avaliação da usabilidade requer um número mínimo de 05 usuários para ser considerada válida, entretanto 07 usuários fizeram uso da ferramenta e preencheram o questionário relatando sua experiência na interação com a aplicação.

As 10 primeiras questões do questionário avaliam a usabilidade da aplicação de sob a perspectiva da interação humano computador e são capazes de identificar 03 heurísticas de Nielsen em seu contexto: a facilidade no aprendizado está implícita nas questões 3, 4, 7 e 10, a eficiência é identificada nas questões 5, 6 e 8 e as questões 1, 2 e 9 se referem à satisfação do usuário (TENÓRIO et al., 2011). As últimas três questões dizem respeito ao nível de satisfação que concerne a interface do sistema e dizem mais sobre questões ergonômicas dentro do sistema, ao passo que se referem à carga cognitiva empregada na utilização da mesma. No decorrer desta seção se buscou explicar de forma mais detalhada a avaliação de cada um deles.

\subsection{AVALIAÇÃO DOS IDOSOS}

De um modo geral, os idosos se mostraram felizes com a utilização do protótipo no sentido de que algo foi pensado com um olhar voltado para eles e também segundo alguns relatos, a aula foi diferente, intuitiva e divertida. Durante a observação foi possível identificar os níveis de dificuldade enfrentado por cada um deles, um pouco devido à idade avançada e outro pelo pouco contato com aplicações deste tipo.

A primeira avaliadora, que no estudo foi nomeada 01 é uma professora aposentada com 79 anos de idade atribuiu um índice de 77,5\% de satisfação no uso do protótipo e 93\% de satisfação na avaliação da interface. Ela testou os três jogos e obteve sucesso na conclusão do jogo da memória e do labirinto, porém não conseguiu concluir o jogo dos oito. Ao final da experiência ela admitiu estar com preguiça de pensar para resolver o desafio e afirmou a 
importância de aplicações desta natureza para a pessoa idosa. De modo geral se sentiu muito confortável na interação com a ferramenta.

A usuária seguinte, denominada 02 é uma administradora do lar com idade aproximada de 60 anos e que apresentou dificuldades no uso dos jogos. Ela solicitou explicações várias vezes, mas ao final conseguiu realizar todas as atividades propostas. Ela ficou extremamente empolgada por ter conseguido concluir todos os jogos, embora não conhecesse a dinâmica de nenhum deles. Seu índice de usabilidade foi de 65\% e avaliação da interface foi de 93\%. Ela relatou ter pouco contato com a tecnologia e que nunca havia jogado jogos como os que foram propostos durante a atividade, contudo se mostrou motivada e grata pela experiência.

O próximo avaliador, aqui denominado com 03 é um senhor de aproximadamente 69 anos utilizou o protótipo com extrema tranquilidade e bastou uma breve explicação para que ele desse continuidade no uso do sistema e na atividade envolvendo os jogos. O mesmo se mostrou extremamente satisfeito durante a atividade e principalmente por ver jogos de sua infância no formato digital. Ele atribui grau máximo de satisfação (100\%) tanto na usabilidade como na interface.

A usuária denominada no estudo de 04 é uma jovem senhora de 50 anos. Em conversa durante a aula, ela relatou que tem dificuldades em utilizar tecnologias, e não as usa com tanta frequência. Ela concluiu o jogo da memória e do labirinto rapidamente, porém não conseguiu resolver o desafio proposto pelo jogo dos oito. Seu grau de satisfação do questionário SUS foi de $80 \%$ e na avaliação da interface o índice obtido foi de 93\%.

A avaliadora denominada 05 é uma senhora de 57 anos que deixou clara não gostar de jogos de raciocínio e ser resistente a tecnologia. Apesar destas afirmações ela concluiu todos os jogos e gostou do uso da ferramenta. Seu índice de usabilidade foi de 82,5\% e o grau de satisfação da interface foi de $80 \%$.

A avaliadora seguinte, que foi chamada de 06 é uma senhora de aproximadamente 65 anos, servidora pública estadual aposentada e que trabalhou durante muitos anos utilizando tecnologias. Ela se mostrou extremamente empolgada em utilizar o sistema e não apresentou 
dificuldade e navegar pelas diferentes telas. Seu índice de usabilidade na escala SUS foi de $87,5 \%$ e o grau de satisfação da interface foi de $87 \%$.

A última avaliadora, denominada no estudo como 07 é uma senhora de aproximadamente 65 anos que se mostrou muito motivada com o uso da ferramenta. Ela conseguiu concluir os três jogos, o último com mais dificuldade mas deixou bem clara a sua opinião sobre a relevância de se projetar esse tipo de site para a população idosa. Seu índice de satisfação do questionário SUS foi de $90 \%$ e a avaliação da interface foi de $100 \%$ de satisfação.

Analisando as heurísticas de Nielsen identificadas nas questões do questionário SUS, a maior dificuldade apresentada pelos usuários de acordo com as respostas ao questionário foi a facilidade de aprendizado, acredita-se que isso se deve muito ao fato deles terem pouco acesso a jogos digitais e também por que alguns não conheciam tais dinâmicas. O Gráfico 1 permite uma melhor visualização.

Gráfico 1: Análise Heurística.

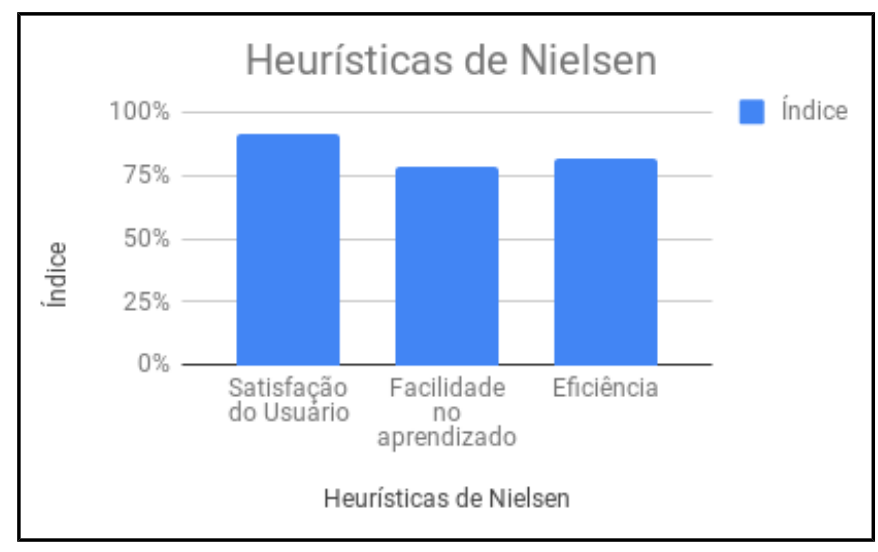

Fonte: Dados primários (2017).

Sob o ponto de vista da análise das questões do questionário SUS o protótipo obteve um grau de usabilidade de 83\% enquanto a interface obteve um grau de satisfação médio de 93\%. Outro fato identificado foi a dificuldade de alguns avaliadores em interpretar as questões do questionário SUS, o que pode ter interferido no resultado final da avaliação ou pode indicar uma diferença entre a real satisfação de cada um sobre o protótipo e as alternativas assinaladas no questionário. Tal constatação também aponta uma necessidade futura de 
adaptar as mesmas com termos mais simples que facilitem o entendimento dos usuários. Juntamente aos resultados individuais, foi feito um cálculo da média de todos os usuários e que indica os índices finais de usabilidade e satisfação.

A avaliação da usabilidade feita pelo principal público-alvo do estudo permitiu prospectar melhorias a serem implementadas no protótipo antes de disponibilizá-los não somente para pessoas portadoras de neurodegeneração mas também para idosos como forma de exercitar a memória e auxiliando-os na aquisição de reserva cognitiva no intuito de prevenir a incidência de transtornos desta natureza. A análise dos resultados gerou sugestões de melhoria importantes no refinamento da aplicação e possibilitará entregar um sistema de qualidade ao usuário final.

\section{CONSIDERAÇÕES FINAIS}

O estudo se mostrou de grande importância no contexto da inclusão digital, da usabilidade e da importância para projetar soluções assistivas sob a perspectiva de cada limitação, neste caso, da pessoa idosa. De acordo com os resultados obtidos, o protótipo obteve alto grau de usabilidade tendo em vista o estudo de Bangor et al. (2009) que afirma que sistemas com índice de usabilidade acima de 70\% representam sistemas altamente usáveis. Avaliar a usabilidade utilizando o público-alvo permitiu observar o olhar crítico das pessoas que estarão diretamente envolvidas na utilização do protótipo.

No que diz respeito a interface os usuários entenderam a mesma como agradável com um layout atrativo e familiar que permitiu uma fácil navegação, já no que se refere aos jogos, acredita-se que foi uma experiência enriquecedora do ponto de vista do estímulo do raciocínio reiterando a ideia da importância do exercício da mente através das mais diversas formas, neste caso por meio de jogos sérios. Outro fator observado é a motivação observada nos idosos com o uso da ferramenta por perceberem que as mais diversas áreas de ciência estão engajadas em promover a prevenção, tratamento e melhoria da qualidade de vida deles.

A análise dos resultados permitiu prospectar futuras melhorias, dentre elas é possível destacar a usabilidade no sentido empregar recursos que permitam que o sistema fique ainda mais interativo, facilitando ainda mais o aprendizado dos usuários. Outro aspecto é a inserção de mais jogos voltados para a prática do treino cognitivo e dificultar os existentes inserindo 
níveis. Destacou-se também a necessidade de adaptar as questões do questionário SUS à linguagem dos idosos, com termos de entendimento mais fácil até mesmo para garantir uma maior acurácia entre a real opinião dos avaliadores e o que foi assinalado na ferramenta de avaliação.

É indispensável que se estude e conheça as condições de vida e de saúde dos mais diversos grupos populacionais de forma a planejar e entregar qualidade de vida aos mesmos (LEON, 2004). Por fim, acredita-se que um dos grandes desafios da sociedade contemporânea será descobrir formas de envelhecer evitando o aparecimento desses sintomas que hoje acometem uma grande fatia da população idosa.

\section{REFERÊNCIAS}

American Psychiatric Association. Diagnostic and statistical 4. Manual of mental disorders. 4th ed. Washington: APA; 1994.

ARAÚJO, M. C. C., FAÇANHA, A. R., DARIN, T. G. R. Um Estudo das Recomendações de Acessibilidade para Audiogames Móveis. XIV Simpósio Brasileiro de acessibilidade. 2010. Disponível em: <https://goo.gl/Cu2qhy >. Acesso 03/11/2017.

AVILA, R., MIOTTO, E. Funções executivas no envelhecimento normal e na doença de Alzheimer. Jornal Brasileiro de Psiquiatria. v.52, p.53 - 62, 2003. Disponível em: <https:/goo.gl/6TUaWm >. Acesso em: 28/08/2017.

BANGOR, A., KORTUM, P. e MILLER, J. Determining What Individual SUS Score Mean. Adding an Adjective Rating Scale. Journal Of Usability Studies, v.4, m.3, p. 114-123, maio 2009.

BEYER, H., HOLTZBLATT, K. Contextual Design. Interactions. January and February 1999. Pg. 32:42.

BRITO, W. D., ZANELATO, A. P. A. A inovação da Web com HTLM5. 2015. Disponível em:

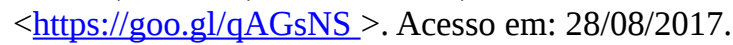

BOUCINHA, R. M., TAROUCO, L. M. R. Avaliação de Ambiente Virtual de Aprendizagem com o uso do SUS - System Usability Scale. CINTED-UFRGS. Novas Tecnologias na Educação. V. 11 No 3, dezembro, 2013.

CHAVES, M. L. F., GODINHO, C. C., PORTO, C. S., MANSUR, L., CARTHERY, M. T., GOULART, M. T., YASSUDA, M. S., BEATO, R. Doença de Alzheimer. Avaliação cognitiva, comportamental e funcional. Dement Neuropsychol 2011 June; 5 (Suppl 1) 21-33.

CORRÊA, A. G. D., ASSIS, G. A., LOPES, R. de D. 2008. GenVirtual. “Um Jogo Musical para Reabilitação de Indivíduos com Necessidades Especiais”, Revista Brasileira de Informática na Educação, vol.16, n. 1. COSTA R. M. E., CARVALHO L. A. V. 2005. O uso de jogos digitais na Reabilitação Cognitiva, In: Workshop de Jogos Digitais na Educação, Minas Gerais, p. 19-21.

ENGHOLM, J. H. Engenharia de software na prática. São Paulo. 2010. 
FORNARI, L. H. T., GARCIA, L. P., HILBIG, A., FERNANDEZ, L. L. As diversas faces da síndrome demencial: como diagnosticar clinicamente? Scientia Medica (Porto Alegre) 2010; volume 20, número 2, p. 185-193.

HAMDAM, A. C. Avaliação Neuropsicológica na doença de Alzheimer e no comprometimento cognitivo leve. Psicol. Argum. 2008 jul./set., 26(54), 183-192. Disponível em: <https://goo.gl/zVJUyc >. Acesso em: 21/08/2017.

INSTITUTO BRASILEIRO DE GEOGRAFIA E ESTATÍSTICA. IBGE. Tábuas completas de mortalidade 2010. Disponível em: <https://goo.gl/pjuRj5 >. Acesso em: 10/09/2017.

LEITE A. Como usar jogos com pessoas com a doença de Alzheimer? 2014 Disponível em: $<$ https://goo.gl/cL7LJW >. Acesso em 01/09/2017.

LEÓN, M.E. dos S. SIG na saúde pública - Estudo de caso: mortalidade infantil em Dom Pedrito/Rs. 2004. Disponível em: <https://goo.gl/1cnNWA > . Acesso em 10/11/2017.

LÉVY, P. Cibercultura. São Paulo: Editora 34, 1999.

MACHADO, F. Análise e gestão de requisitos de software: onde nascem os sistemas. 2ª ed. São Paulo. 2014.

MAFRA, S. C. T., Elaboração de Check List para desenvolvimento de projetos eficientes de cozinhas a partir de mapas mentais e escala Likert. 1999. Disponível em: <https://goo.gl/zCXvNw >. Acesso em: 12/09/2017.

MENDES,L.S. Análise ergonômica da situação dos idosos pedestres em relação à sinalização de Copacabana. 2008. Rio de Janeiro: PUCRJ - Departamento de Artes e Design.

NIELSEN, J., LORANGER, H. Usabilidade na Web. 2007. Rio de Janeiro: Elsiever.

NIELSEN, J. Usability Engineering, Morgan Kaufman, Inc. San Francisco, 1993.

NIELSEN, J. Usability 101: Introduction to usability 2003. Disponível em:

<https://goo.gl/1J65su >. Acesso em: 13/07/2017.

OLIVEIRA, A. de. Tecnologia Assistiva - Um tema em ascenção. Aplicação de recursos em tecnologia assistiva na educação. I Seminário de Pesquisa, Pós-Graduação e Inovação. Araranguá - 2015. Disponível em:

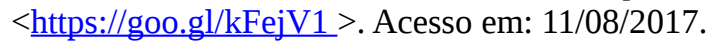

PAZ, F. J., LEITE, M.C.D. Avaliação da usabilidade do aplicativo comunique-se: um app para pacientes que sofreram AVC. 14ª Jornada de Pós-Graduação e Pesquisa. Bagé - RS . Outubro, 2017.

PRADO, E.F. do. Introdução ao desenvolvimento de games com GWT e HTML5. XI SBGames - Brasília DF - Brazil, November 2nd - 4th, 2012.

PRESSMAN, R. S. Engenharia de Software - Uma Abordagem Profissional. 7. ed. Porto Alegre. AMGH. 2011.

PRODANOV, C. C., FREITAS, E. C. de. METODOLOGIA DO TRABALHO CIENTÍFICO: Métodos e Técnicas da Pesquisa e do Trabalho Acadêmico. 2013. $2^{\text {a }}$ edição. Universidade Feevale - Novo Hamburgo Rio Grande do Sul - Brasil. Disponível em: <https://goo.gl/3Ph4Y4 >. Acesso em 28/08/2017.

RIEDER R., ZANELATTO E., BRANCHER J. 2005. Observação e análise da aplicação de jogos educacionais bidimensionais em um ambiente aberto. Journal of Computer Science, vol 4, n. 2, p. 63-71. 
RIOS, G. A., JUNIOR, K. S., VIGENTIN, U. D., MAGRI, C. M. Audiodescrição e inclusão na educação a distância: experiência do núcleo de educação a distância da UNESP. Jorsen Volume 16 Issue S1. August 2016. Pages 236-240.

ROCHA, P., ALVES, L., NERY J. Jogos digitais e reabilitação neuropsicológica: delineando novas mídias. 2014. I Seminário Tecnologias Aplicadas Educação e Saúde. UNEB, Campus I,Salvador - BA. Disponível em : <https://goo.gl/5vfWud >. Acesso em 24/08/2017.

SOMMERVILLE, I. Engenharia de Software. $9^{\text {a }}$ ed. 2012.

SOUZA, D., FRANÇA, F., CAMPOS T. Teste de labirinto: instrumento de análise na aquisição de uma habilidade motora. Brazilian Journal of Physical Therapy. 2006; 10:335-60.

TENÓRIO, J. M., SDEPANIAN, V. L., PISA, I.T., COHRS F. M., MARIN H. F. Desenvolvimento e Avaliação de um Protocolo Eletrônico para Atendimento e Monitoramento do Paciente com Doença Celíaca. Rev. Inform. Teór. Aplic. 17(2) V.2 p, 10-20,2011.

WANATABE, M. K. F et al. Terapia Ocupacional e o uso do computador como recurso terapêutico. Acta Fisiátrica 10(1): 17 - 20, 2003. Disponível em: < https://goo.gl/DGnPvy > . Acesso em 11/08/2017.

WHITLOCK, L.A., MCLAUGHLIN A. C., ALLAIRE J. C. Individual differences in response to cognitive training: using a multi-modal, attentionally demanding game-based intervention for older adults. Computers in human behavior. 2012; 28(4): 1091-96.

World Health Organization. WHO Guidelines on Health-Related Rehabilitation, Rehabilitation Guidelines.

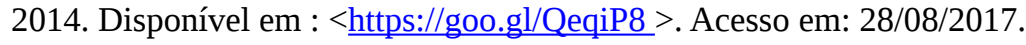

W3C (2014). Web content accessibility guidelines (wcag) 2.0. Disponível em: <https://goo.gl/1HmrXL >. Acesso em : 25/08/2017.

XAVIER, A. et al. Oficina da Lembrança: Ambiente de Inclusão Digital e Tele-Reabilitação Cognitiva para Idosos, Portadores de Doença de Alzheimer e Outros Distúrbios Cognitivos. 2016. Disponível em: <https://goo.gl/W2GL25 >. Acesso em: 12/08/2017. 